\title{
Time series of stock price and of two fractal overlap: Anticipating market crashes?
}

\author{
Bikas K. Chakrabarti ${ }^{1}$, Arnab Chatterjee ${ }^{2}$ and Pratip Bhattacharyya ${ }^{3}$ \\ 1 Theoretical Condensed Matter Physics Division and Centre for Applied \\ Mathematics and Computational Science, Saha Institute of Nuclear Physics, \\ Block-AF, Sector-I Bidhannagar, Kolkata-700064, India. \\ bikas@cmp.saha.ernet.in \\ 2 arnab@cmp.saha.ernet.in \\ 3 pratip@cmp.saha.ernet.in
}

We find prominent similarities in the features of the time series for the overlap of two Cantor sets when one set moves with uniform relative velocity over the other and time series of stock prices. An anticipation method for some of the crashes have been proposed here, based on these observations.

\section{Introduction}

Capturing dynamical patterns of stock prices are major challenges both epistemologically as well as financially [1]. The statistical properties of their (time) variations or fluctuations [1] are now well studied and characterized (with established fractal properties), but are not very useful for studying and anticipating their dynamics in the market. Noting that a single fractal gives essentially a time averaged picture, a minimal two-fractal overlap time series model was introduced $[2,3,4]$.

\section{The model}

We consider first the time series $O(t)$ of the overlap sets of two identical fractals $[4,5]$, as one slides over the other with uniform velocity. Let us consider two regular cantor sets at finite generation $n$. As one set slides over the other, the overlap set changes. The total overlap $O(t)$ at any instant $t$ changes with time (see Fig. 1(a)). In Fig. 1(b) we show the behavior of the cumulative overlap [4] $Q^{o}(t)=\int_{0}^{t} O(\tilde{t}) d \tilde{t}$. This curve, for sets with generation $n=4$, is approximately a straight line [4] with slope $(16 / 5)^{4}$. In general, this curve approaches a strict straight line in the limit $a \rightarrow \infty$, asymptotically, where the overlap set comes from the Cantor sets formed of $a-1$ blocks, taking away 
the central block, giving dimension of the Cantor sets equal to $\ln (a-1) / \ln a$. The cumulative curve is then almost a straight line and has then a slope $\left[(a-1)^{2} / a\right]^{n}$ for sets of generation $n$. If one defines a 'crash' occurring at time $t_{i}$ when $O\left(t_{i}\right)-O\left(t_{i+1}\right) \geq \Delta$ (a preassigned large value) and one redefines the zero of the scale at each $t_{i}$, then the behavior of the cumulative overlap $Q_{i}^{o}(t)=\int_{t_{i-1}}^{t} O(\tilde{t}) d \tilde{t}, \tilde{t} \leq t_{i}$, has got the peak value 'quantization' as shown in Fig. 1(c). The reason is obvious. This justifies the simple thumb rule: one can simply count the cumulative $Q_{i}^{o}(t)$ of the overlaps since the last 'crash' or 'shock' at $t_{i-1}$ and if the value exceeds the minimum value $\left(q_{o}\right)$, one can safely extrapolate linearly and expect growth upto $\alpha q_{o}$ here and face a 'crash' or overlap greater than $\Delta$ (=150 in Fig. 1). If nothing happens there, one can again wait upto a time until which the cumulative grows upto $\alpha^{2} q_{o}$ and feel a 'crash' and so on ( $\alpha=5$ in the set considered in Fig. 1).

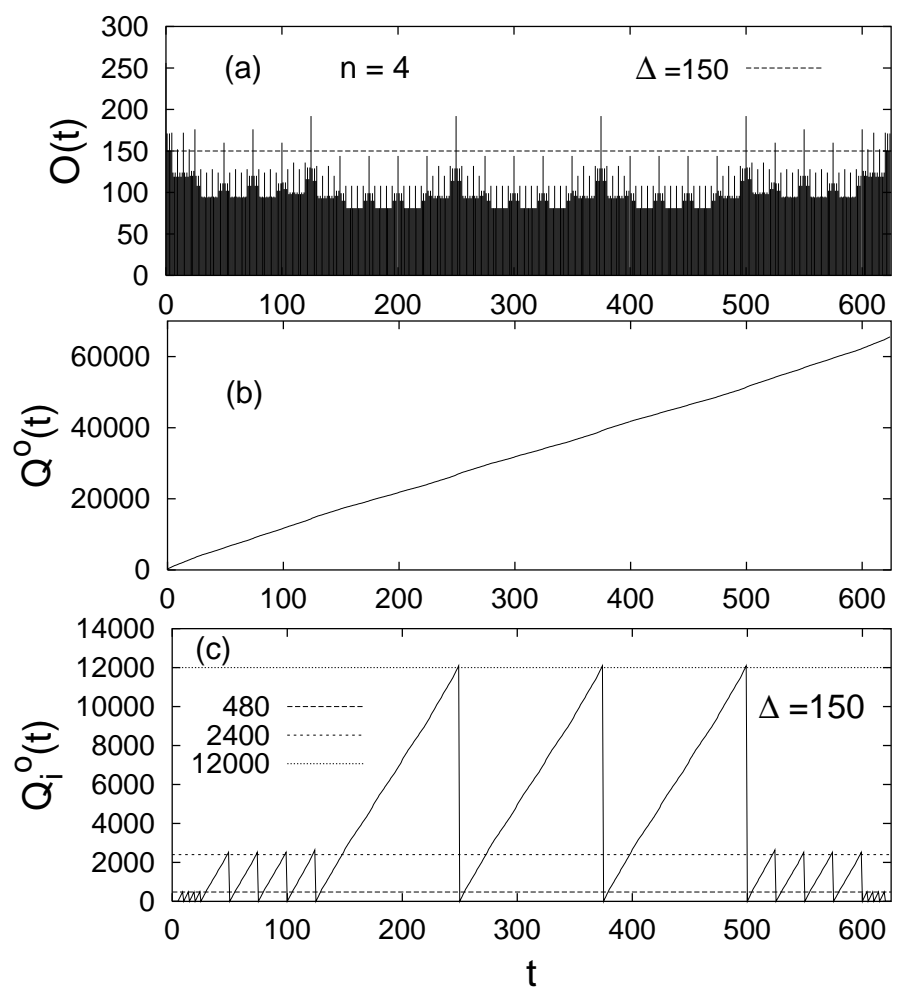

Fig. 1. (a) The time series data of overlap size $O(t)$ for a regular Cantor set of dimension $\ln 4 / \ln 5$ at generation $n=4$. (b) Cumulative overlap $Q^{\circ}(t)$ and (c) the variation of the cumulative overlap $Q_{i}^{o}(t)$ for the same series, where $Q$ is reset to zero after any big event of size greater than $\Delta=150$. 
We now consider some typical stock price time-series data, available in the internet [6]. In Fig. 2(a), we show that the daily stock price $S(t)$ variations for about 10 years (daily closing price of the 'industrial index') from January 1966 to December 1979 (3505 trading days). The cumulative $Q^{s}(t)=\int_{0}^{t} S(t) d t$ has again a straight line variation with time $t$ (Fig. 2(b)). We then define the major shock by identifying those variations when $\delta S(t)$ of the prices in successive days exceeded a preassigned value $\Delta$ (Fig. 2(c)). The variation of $Q_{i}^{s}(t)=\int_{t_{i-1}}^{t_{i}} S(\tilde{t}) d \tilde{t}$ where $t_{i}$ are the times when $\delta S\left(t_{i}\right) \leq-1$ show similar geometric series like peak values (see Fig. 2(d)).
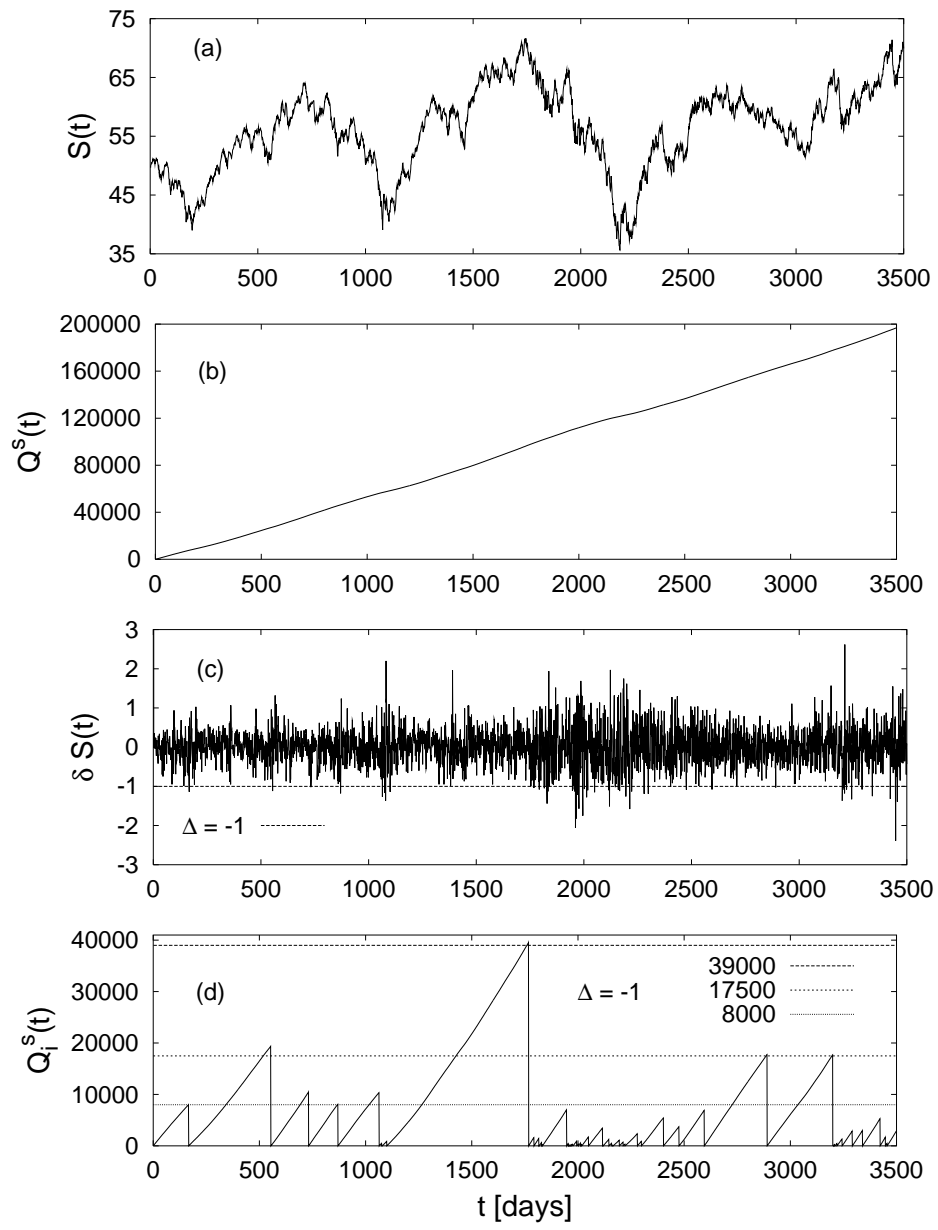

Fig. 2. Data from New York Stock Exchange from January 1966 to December 1979: industrial index [6]: (a) Daily closing index $S(t)$ (b) integrated $Q^{s}(t)$, (c) daily changes $\delta S(t)$ of the index $S(t)$ defined as $\delta S(t)=S(t+1)-S(t)$, and (d) behavior of $Q_{i}^{s}(t)$ where $\delta S\left(t_{i}\right)>\Delta$. Here, $\Delta=-1.0$ as shown in (c) by the dotted line. 
A simple 'anticipation strategy' for some of the crashes may be as follows: If the cumulative $Q_{i}^{s}(t)$ since the last crash has grown beyond $q_{0} \simeq 8000$ here, wait until it grows (linearly with time) until about $17,500\left(\simeq 2.2 q_{0}\right)$ and expect a crash there. If nothing happens, then wait until $Q_{i}^{s}(t)$ grows (again linearly with time $)$ to a value of the order of $39,000\left(\simeq(2.2)^{2} q_{0}\right)$ and expect a crash, and so on.

\section{Summary}

The features of the time series for the overlap of two Cantor sets when one set moves with uniform relative velocity over the other looks somewhat similar to the time series of stock prices. We analyze both and explore the possibilities of anticipating a large (change in Cantor set) overlap or a large change in stock price. An anticipation method for some of the crashes has been proposed here, based on these observations.

\section{References}

1. Sornette D (2003) Why Stock Markets Crash? Princeton Univ. Press, Princeton; Mantegna RN, Stanley HE (1999) Introduction to Econophysics. Cambridge Univ. Press, Cambridge

2. Chakrabarti BK, Stinchcombe RB (1999) Physica A 270:27-34

3. Pradhan S, Chakrabarti BK, Ray P, Dey MK (2003) Phys. Scr. T106:77-81

4. Pradhan S, Chaudhuri P, Chakrabarti BK (2004) in Continuum Models and Discrete Systems, Ed. Bergman DJ, Inan E, Nato Sc. Series, Kluwer Academic Publishers, Dordrecht, pp.245-250; cond-mat/0307735

5. Bhattacharyya P (2005) Physica A 348:199-215

6. NYSE Daily Index Closes from http://www.unifr.ch/econophysics/. 